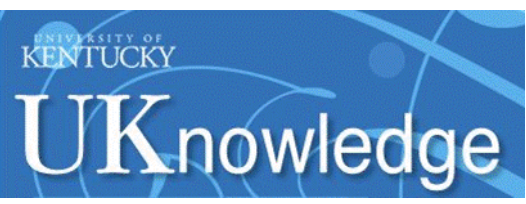

University of Kentucky

UKnowledge

\title{
Translating Pharmacogenetics to Clinical Practice: Do Cytochrome P450 2D6 Ultrarapid Metabolizers Need Higher Atomoxetine Doses?
}

Jose de Leon

University of Kentucky, jdeleon@uky.edu

Follow this and additional works at: https://uknowledge.uky.edu/psychiatry_facpub

Part of the Psychiatry and Psychology Commons

Right click to open a feedback form in a new tab to let us know how this document benefits you.

\section{Repository Citation}

de Leon, Jose, "Translating Pharmacogenetics to Clinical Practice: Do Cytochrome P450 2D6 Ultrarapid Metabolizers Need Higher Atomoxetine Doses?" (2015). Psychiatry Faculty Publications. 32.

https://uknowledge.uky.edu/psychiatry_facpub/32

This Article is brought to you for free and open access by the Psychiatry at UKnowledge. It has been accepted for inclusion in Psychiatry Faculty Publications by an authorized administrator of UKnowledge. For more information, please contact UKnowledge@lsv.uky.edu. 


\section{Translating Pharmacogenetics to Clinical Practice: Do Cytochrome P450 2D6 Ultrarapid Metabolizers Need Higher Atomoxetine Doses?}

Digital Object Identifier (DOI)

http://dx.doi.org/10.1016/j.jaac.2015.04.003

Notes/Citation Information

Published in Journal of the American Academy of Child \& Adolescent Psychiatry, v. 54, no. 7, p. 532-534.

(C) 2015 American Academy of Child and Adolescent Psychiatry

This manuscript version is made available under the CC-BY-NC-ND 4.0 license

http://creativecommons.org/licenses/by-nc-nd/4.0/ 
Translating Pharmacogenetics to Clinical Practice:

Do Cytochrome P450 2D6 Ultrarapid Metabolizers Need Higher Atomoxetine Doses?

Jose de Leon, MD

(C) 2015 American Academy of Child and Adolescent Psychiatry

This manuscript version is made available under the CC-BY-NC-ND 4.0 license

http://creativecommons.org/licenses/by-nc-nd/4.0/ 
Words in text: 1,499 words

References: 5

Table: 1

\section{TRANSLATIONS:}

Translating Pharmacogenetics to Clinical Practice:

Do CYP2D6 Ultrarapid Metabolizers Need Higher Atomoxetine Doses?

Jose de Leon, M.D.*

*University of Kentucky Mental Health Research Center at Eastern State Hospital, Lexington, KY, and Psychiatry and Neurosciences Research Group (CTS-549), Institute of Neurosciences, University of Granada, Granada, Spain, and Biomedical Research Centre in Mental Health Net (CIBERSAM), Santiago Apóstol Hospital, University of the Basque Country, Vitoria, Spain.

Running title: Atomoxetine and CYP2D6

Corresponding author: Jose de Leon, M.D., UK Mental Health Research Center at Eastern State Hospital, 1350 Bull Lea Road, Lexington, KY 40511. Phone (859) 246-8440. Fax (859) 246-8446. e-mail: jdeleon@uky.edu

Disclosure of interest: No commercial organizations had any role in the completion or publication of this article. Dr. de Leon reports no biomedical financial interests or potential conflicts of interest in the last 2 years and in the foreseeable future. This article was completed without any external funding.

Acknowledgements: The author thanks Lorraine Maw, M.A., at the UK Mental Health Research Center, for editorial assistance. The author is grateful to the reviewers who helped him improve this article. 


\section{EIGHTEEN YEARS OF PHARMACOGENETICS}

Pharmacogenetics and personalized prescription have long and overlapping histories but they were probably born with the DNA microarray technology which allows parallel genetic testing. ${ }^{1}$ In 1997 , Science defined "personalized prescription" as "tailoring drugs to a patient's genetic makeup" and predicted that personalized prescription would "soon" reach clinical practice. The end of the race for the Human Genome in 2000 led to further hype. The most widely circulated US news magazine, Time, predicted that the generalized use of personalized prescription would begin in 2015, while JAMA predicted 2020. Now that it's 2015, are we any closer to using pharmacogenetic testing to guide our pharmacological treatments? The answer is obviously, "No". "No" both in child psychiatry and medicine in general.

How is it possible that personalized prescription has not reached adulthood in 2015 following those rosy predictions? The answer lies in two facts: 1) the process of implementing pharmacogenetic testing under the auspices of the Food and Drug Administration (FDA) was flawed, and 2) personalizing prescription is even more complex than pharmacogenetic testing, as the story of atomoxetine demonstrates.

\section{THE FDA AND PHARMACOGENETICS}

In the 1990s, the FDA implemented guidelines to encourage the introduction of pharmacogenetic testing in the clinical environment and defined the cytochrome P450 2D6 (CYP2D6) as a "valid biomarker." The pharmaceutical companies felt threatened; new drugs would be subjected to the complications of pharmacogenetic testing and/or potentially smaller markets. CYP2D6 is the main metabolic enzyme for many psychiatric drugs but, due to the unpredictable twists of evolution, it is absent in 7\% of Caucasians. They have two non-functional CYP2D6 alleles and are called poor metabolizers (PMs). On the other hand, approximately $1.5 \%$ of US Caucasians and African-Americans have 3 or more active alleles, and produce more than normal quantities of CYP2D6 in their livers. They are called ultrarapid metabolizers (UMs). To avoid problems, the pharmaceutical companies decided to eliminate 
from their pipelines drugs heavily dependent on CYP2D6 for their metabolism; atomoxetine was one of the last ones marketed. Its manufacturer had to meet the FDA guidelines and demonstrate that atomoxetine was safe for CYP2D6 PMs.

\section{COMPLEXITIES OF PERSONALIZED PRESCRIPTION AND PHARMACOGENETICS}

FDA guidelines do not sufficiently account for the complexity of the clinical environment, as PM status can be explained by different mechanisms. Obviously, genetics may cause CYP2D6 PM status. Secondly, clinicians can produce PM status by prescribing powerful CYP2D6 inhibitors, such as paroxetine or fluoxetine, which can cause patients with normal CYP2D6 activity, called extensive metabolizers (EMs), to behave as if they have no CYP2D6 activity. This exemplifies CYP2D6 PM status explained by environmental factors. Lastly, for some CYP2D6 drugs partly eliminated by the kidney, such as risperidone, renal insufficiency can account for individuals who look like PMs; in this case a personal factor has produced a CYP2D6 PM status.

Therefore, personalized prescription should include genetic as well as environmental (e.g., comedications, herbal medicines, smoking, foods, beverages) and personal (e.g., age, gender, ethnicity, illness) variables. ${ }^{2}$ To further complicate things, personalized prescription can be expressed as personalizing dosing and/or drug selection, and should consider pharmacokinetic and pharmacodynamic mechanisms which influence both the safety and efficacy of drugs. In the case of atomoxetine, one of these pieces in the complex puzzle of personalized prescribing was ignored, namely, the width of its therapeutic index (or window). ${ }^{3}$

The best way of implementing personalized dosing ${ }^{2}$ is by combining pharmacogenetics with blood levels, which pharmacologists call therapeutic drug monitoring (TDM). Unfortunately for atomoxetine, its data is hampered since 1) all the related pharmacogenetic studies focused on CYP2D6 PMs and safety during the randomized clinical trials (RCTs), as required by the FDA; and 2) the published TDM data focuses on peak concentrations, which are harder to interpret than the steady-state trough levels studied by the traditional TDM studies in the clinical environment. 
ATOMOXETINE AND CYP2D6 PMs

In pharmacokinetic studies, at steady state, after twice-daily dosing, the mean steady-state plasma concentrations were approximately 10-fold higher in CYP2D6 PMs than in CYP2D6 EMs. However, these pharmacokinetic differences had no relevance to adverse drug reactions (ADRs) during the RCTs. The most comprehensive ADR report ${ }^{4}$ compared 87 CYP2D6 PMs and 1239 CYP2D6 EMs. Despite a lower mean dose in PMs, they demonstrated, when compared with EMs, marginally better efficacy in response rate $(85 \%$ vs. $82 \%, p=0.56$, odds ratio, OR calculated with article data by this author $=1.2,95 \%$ confidence interval, CI, 0.79-2.1) and comparable ADR profiles with a difference in discontinuation due to ADRs of $6 \%$ vs. $2 \%(\mathrm{OR}=2.5,1.1-5.6)$, which did not reach significance $(p=0.08)$.

Atomoxetine was marketed and the US prescribing information (or package insert) recommends lower doses for CYP2D6 PMs or those taking CYP2D6 inhibitors, but it makes no comment on the need for CYP2D6 genotyping before prescribing atomoxetine. Thus, child psychiatrists do not need to worry about CYP2D6 genotyping when prescribing atomoxetine.

\section{ATOMOXETINE AND CYP2D6 UMs}

Atomoxetine follows linear kinetics. This means that the relationship between concentration doses is stable and very predictable. The pharmaceutical company should have focused not on atomoxetine ADRs in CYP2D6 PMs, but on the lack of efficacy in CYP2D6 UMs. As CYP2D6 PMs have a 10-fold increase in atomoxetine levels with no relevant increase in ADRs, this proves that atomoxetine is a very safe drug with a wide therapeutic index. Prior pharmacological experience indicates that in drugs with a wide therapeutic index, one needs to focus on UMs, who may not have sufficient drug levels at the usual recommended dosages. ${ }^{3}$

Table 1 describes the response rate at the endpoint of the acute treatment phase ${ }^{5}$ and uses these response rates to make predictions. To increase the response rate of EMs in the acute phase from the $60 \%$ obtained to the $80 \%$ seen in CYP2D6 PMs, one needs to get the same atomoxetine levels in CYP2D6 EMs as in PMs. As atomoxetine follows linear kinetics, one would have to increase the EM dose 10-fold 
to get approximately the same mean plasma concentration as the PMs in the RCTs. Table 1 indicates that up to $20 \%$ of CYP2D6 EMs were underdosed in the RCTs, which may have contributed to a $9 \%$ increase in dropouts due to lack of efficacy in CYP2D6 EMs.

In the RCTs, CYP2D6 UMs were included in the CYP2D6 EM group and probably were heavily overrepresented in the group lacking efficacy. Assuming that atomoxetine follows linear kinetics and that, as in other CYP2D6 drugs, UMs had an increase in metabolism by at least a factor of 2 when compared with EMs, one needs to conclude that, during the RCTs, CYP2D6 UMs probably had less than half the atomoxetine levels as EMs, and approximately 20 times less than CYP2D6 PMs. If this is correct, UM doses 20 times higher than used in the RCTs would provide the 80\% response rate seen in CYP2D6 PMs.

New studies using CYP2D6 genotyping and TDM are needed to establish whether or not higher atomoxetine doses are required for CYP2D6 UMs (1.5\% in US Caucasians and 2.0\% in AfricanAmericans) and possibly in CYP2D6 EMs with $\geq 2$ more active alleles (respectively, 36.4\% and 18.3\%) who may not respond to recommended doses. Unfortunately, the independent investigators testing this hypothesis would have to start from scratch, as CYP2D6 UM response information is absent and atomoxetine TDM data is limited. The company completed a study of two-times higher doses of nonresponders that was negative, but was not guided by TDM or CYP2D6 genotyping. The author proposes that after CYP2D6 genotyping, those subjects with $\geq 2$ active alleles, which in total account for approximately one-third of US individuals, can be treated with much higher atomoxetine doses than those recommended in the package insert, as long as atomoxetine TDM demonstrates safe steady-state trough concentrations similar to those seen in CYP2D6 PMs with recommended doses.

In summary, clinicians may need to be aware that normal atomoxetine doses may not be enough for CYP2D6 $\mathrm{UMs}^{3}$ and possibly for CYP2D6 EMs $\geq 2$ active alleles. As a matter of fact, in 2011 The Royal Dutch Association for the Advancement of Pharmacy's CYP genotyping guidelines concurred that dosing atomoxetine in CYP2D6 UMs is problematic and recommended that clinicians "be alert to reduced efficacy or select alternative drugs." Researchers interested in studying patients who do not respond to 
atomoxetine have the challenge of building a new database by combining the concepts of CYP2D6 genotyping, atomoxetine TDM and wide therapeutic index.

\section{THE DIFICULTIES OF IMPLEMENTING PERSONALIZED MEDICINE}

Atomoxetine exemplifies why personalized medicine and pharmacogenetic testing are not widely used in 2015 by child psychiatrists or physicians in general. Personalizing prescription is a complex pharmacological puzzle that 1) can focus on drug dosing or drug selection; 2) requires combining genetics, environmental and personal variables; and 3) necessitates understanding pharmacokinetic and pharmacodynamic mechanisms in order to predict efficacy and safety. If one focuses on dosing, one also needs to consider the drug's therapeutic index. As a matter of fact, as each patient is a "unique individual" with different drug responses governed by pharmacological laws, each drug may also be a "different individual" governed by its specific pharmacological mechanisms.

\section{REFERENCES}

1. de Leon J. Evidence-based medicine versus personalized medicine: are they enemies? (editorial). J Clin Psychopharmacol 2012;32:153-164.

2. de Leon J. The future (or lack of future) of personalized prescription in psychiatry. Pharmacol Res 2009;59:81-89.

3. de Leon J. The crucial role of the therapeutic window in understanding the clinical relevance of the poor versus the ultrarapid metabolizer phenotypes in subjects taking drugs metabolized by CYP2D6 or CYP2C19. J Clin Psychopharmacol 2007;27:241-245.

4. Trzepacz PT, Williams DW, Feldman PD et al. CYP2D6 metabolizer status and atomoxetine dosing in children and adolescents with ADHD. Eur Neuropsychopharmacol 2008;18:79-86.

5. Michelson D, Read HA, Ruff DD et al. CYP2D6 and clinical response to atomoxetine in children and adolescents with ADHD. J Am Acad Child Adolesc Psychiatry 2007;46:242-251. 
Table 1.Comparing atomoxetine response rate ${ }^{\mathrm{a}}$ of CYP2D6 PMs to EMs

$\underline{\mathrm{RCT}}$ dose

PMs EMs Difference
$\mathrm{N}$

Response rate

$30 \quad 559$

$80 \% \quad 60 \% \quad 20 \%{ }^{b}$

Dropouts due to lack of efficacy $\quad 18 \% \quad 27 \% \quad-9 \%{ }^{\mathrm{c}} \quad 18 \%$

Predictions if dose is 10 times higher EMs

$80 \%$

CI: 95\% confidence interval; EM: extensive metabolizer; PM: poor metabolizer; OR: odds ratio; RCT: randomized clinical trial.

${ }^{a}$ At the endpoint of the acute treatment phase.

${ }^{\mathrm{b}}$ This difference was described as significant $(\mathrm{p}=0.033) .{ }^{5}$ The author estimated an OR comparing PMs vs. EMs of 2.7, CI, 1.35.9 .

${ }^{\mathrm{c}}$ This difference was described as significant $(\mathrm{p}=0.004){ }^{5}$ The OR comparing PMs vs. EMs was 0.57, CI, 0.40-0.79. 